\title{
UPDATED VERSION OF THE SIMPLIFIED MODEL OF INTENSIVELY BLASTED ELECTRIC ARC
}

\author{
Josef SenK*, Ilona LaZnickova, Ivana Jakubova \\ Faculty of Electrical Engineering and Communication, BUT, Technicka 12, 61600 Brno, Czech Republic \\ * corresponding author: senk@feec.vutbr.cz
}

\begin{abstract}
The article deals with an improvement of a simplified model of intensively blasted electric arc burning in argon in an anode channel of a modular-type arc heater. Gradually gained experience in the application of the model with various input data has revealed that some refinement is needed. The contribution focuses especially on the process of searching for an optimum combination of the state variables, which are the exponent determining the arc radius development along the anode channel axis, the current density at the cathode, and the arc temperature at the end of the near-cathode layer. The limiting relations for the state variables are set and discussed. Three constituent parts of the used objective function are analysed and compared. The procedure is demonstrated on a chosen set of experimental data. Further computations with many sets of measured data are necessary to test the designed procedure and to confirm the determination of the state variables' range.
\end{abstract}

KEYWORDS: electric arc, arc heater, argon, modeling.

\section{INTRODUCTION}

For both research and educational purposes, a simplified mathematical model of the intensively blasted electric arc burning in an anode channel of an experimental modular-type arc heater has been designed, applied for the evaluation of experimental results and based on the gradually gained experience, also updated and refined. The main aim of the model is to determine the arc radius $r_{\mathrm{A}}$, the arc temperature $T_{\mathrm{A}}$ and the arc voltage $U_{\mathrm{A}}$ distribution from the experimentally obtained integral data, such as the total voltage $U$, current $I$, the gas flow rate $Q_{\mathrm{m}}$ and power loss $P_{\text {loss }}$ of individual segments of the arc heater found out by calorimetry. The arc heater can be operated with various working gases whose transport and thermodynamic properties must be available for the computation. Here, experiments carried out with argon are discussed.

The core of the model includes the energy and mass conservation laws and Ohm's law [1]. For computations, the arc is divided by a suitable mesh along the anode channel axis and basic equations are rewritten in difference forms. Simplifications are applied, some of them based on the geometry of the device (axial symmetry), properties of the working medium or physical phenomena taking place inside the anode channel (e.g., local thermodynamic equilibrium of the plasma; omission of plasma kinetic energy; only the radial component of the radiative energy flow and only the axial component of the enthalpy flow taken into account; a neglect of the conductive energy term; Mach number Ma constant over the anode channel crosssection). Other simplifications are based on previous calculations, especially the rectangular temperature distribution across the anode channel has been found to be sufficient: in the arc zone $0 \leq r \leq r_{\mathrm{A}}$ the temper- ature is $T_{\mathrm{A}}(r, z)=T_{\mathrm{A}}(z)$ and in the cold zone between the arc and the anode channel wall $r_{\mathrm{A}}<r<r_{\mathrm{C}}$, the gas temperature remains the same as at the input $T_{0}$. Otherwise, some originally applied approximations have been revealed as inappropriate and have been discarded (e.g., previous neglect of near-electrode voltage drops). Related considerations and modifications of the model are described in detail in previous papers of the authors [1, 2].

Recently, two key modifications have been implemented to the model. First, the method of calculation of the arc power loss has been completely changed. Instead of the application of the same power-loss ratio $p_{\text {loss }}$ in each segment of the arc heater, power loss of the arc is now estimated using the theoretically calculated net emission coefficient of argon [3 5]. This way, a very good agreement between the computed and measured distribution of the power loss all along the arc heater anode channel can be reached, but simultaneously, computations have revealed that the previously used constant value of the current density on the cathode does not meet the needs. Thus, the second substantial modification has been made, consisting in expanding the set of state variables to three: the exponent determining the arc radius development along the anode channel axis, the arc temperature at the end of the near-cathode layer, and the current density at the cathode.

The paper focuses especially on the process of searching an optimum combination of the state variables. In the following section, the state variables are characterized and their range and limiting conditions are explained. In Section 3 the obtained values of the used objective function in chosen ranges are illustrated in figures and discussed. In Section 4 , the found candidates are analysed and their mutual relationships are commented. Section 5 concludes the paper. 


\section{The Set of State Variables}

The most important parameter, which plays a leading role in the description of the arc column, is the arc radius and its development along the arc heater anode channel $r_{\mathrm{A}}(z)$. The inside part of the anode channel is not accessible for a direct measurement, so the following formula from [6] is used:

$$
r_{\mathrm{A}}(z)=r_{0}\left[1+\left(\frac{z}{r_{0}}\right)^{1 / n_{\mathrm{r}}}\right]
$$

where $r$ is the radius $(\mathrm{m}), z$ is the axial coordinate (m). Subscript 0 stands for the beginning $z=0$ at the cathode tip. Obviously, there are two parameters defining this function, the exponent $n_{\mathrm{r}}$ and the radius of the cathode spot $r_{0}$.

In previous versions of the model, a priority was given to the choice of the exponent $n_{\mathrm{r}}$ and the solution was sought for around the value of 3 , which is given by other authors for a free cylindrically symmetric beam 6]. A similar value of $n_{\mathrm{r}}$ was also confirmed by an approximate calculation for the output crosssection of the anode. The radius of the cathode spot $r_{0}$ was determined from the measured arc current $I$ and the current density at the cathode tip $j_{0}$, which was supposed to be $1 \times 10^{8} \mathrm{Am}^{-2}$ for currents up to $2.16 \mathrm{kA}$ [7].

This presumption has been found to be too restrictive. As can be seen in Figure 1, through the radius of the cathode spot $r_{0}$, the current density at the cathode tip $j_{0}$ influences the shape of the arc column along the entire anode channel. Furthermore, it also figures in the computation of the near-cathode layer $s$ as explained in [1]. The close interdependence of mentioned quantities has not been sufficiently considered in the previous versions of the model.

In the updated version of the model, the current density at the cathode tip $j_{0}$ is included in the set of the state variables. As demonstrated in Sec. 3, the results are rather sensitive to this parameter. The choice of the range of the current density at the cathode tip $j_{0}$ relies on experimentally obtained results of other authors. Unfortunately, the current density at the cathode tip $j_{0}$ strongly depends on experimental conditions. With respect to this fact, values from $1 \times 10^{8} \mathrm{~A} \mathrm{~m}^{-2}$ to $(3-4) \times 10^{8} \mathrm{~A} \mathrm{~m}^{-2}$ seem to be reasonable [8, 9].

The arc temperature at the end of the near-cathode layer $T_{\mathrm{A}}(s)$ serves as the third state variable. It must be higher than $6000 \mathrm{~K}$, which is the temperature where conductivity of argon starts to rise. The value of $T_{\mathrm{A}}(s)$ is set as high as possible to reach a steep but smooth increase in $T_{\mathrm{A}}(z)$ dependence, without oscillations.

The vector of the three state variables $\left[n_{\mathrm{r}} ; j_{0} ; T_{\mathrm{A}}(s)\right]$ is sought for when the objective function

$$
\Delta_{\text {tot }}=\sqrt{\Delta_{\text {in }}^{2}+\Delta_{\text {ch }}^{2}+\Delta_{\mathrm{U}}^{2}}
$$

reaches its minimum. The objective function includes three constituent parts. The first two terms express how the computed (subscript c) power losses of the arc in the input segment of the anode channel (subscript in) and in the main segment of the anode channel (subscript $\mathrm{ch}$ ) match the measured (subscript m) power losses of the corresponding segments. In other words, the first two terms show how truly the model can capture the behaviour of the arc in individual inner parts of the anode channel. In the third term $\Delta_{\mathrm{U}}$, it is namely the computed potential increment $U_{\mathrm{A}}\left(z_{\mathrm{L}}\right)$ of the arc between the beginning $z=0$ and the end $z=z_{\mathrm{L}}$ of the anode channel that is crucial. Because the arc potential increment $U_{\mathrm{A}}\left(z_{\mathrm{L}}\right)$ cannot be measured separately, $\Delta_{\mathrm{U}}$ must be judged by comparing the sum of the calculated input power of the arc $U_{\mathrm{Ac}}\left(z_{\mathrm{L}}\right) I_{\mathrm{m}}$, the calculated power loss of the arc-anode attachment $P_{\text {loss as c }}$ and the measured cathode power loss $P_{\text {loss cat } \mathrm{m} \text { against the measured total input power }}$ $U_{\mathrm{m}} I_{\mathrm{m}}$. The constituent parts of the objective function are calculated as follows:

$$
\begin{aligned}
& \Delta_{\text {in }}=\frac{P_{\text {lossin } \mathrm{c}}}{P_{\text {loss in } \mathrm{m}}}-1, \quad \Delta_{\mathrm{ch}}=\frac{P_{\text {loss ch } \mathrm{c}}}{P_{\text {loss ch } \mathrm{m}}}-1, \\
& \Delta_{\mathrm{U}}=\frac{U_{\mathrm{A} \mathrm{c}}\left(z_{\mathrm{L}}\right) I_{\mathrm{m}}+P_{\text {loss cat } \mathrm{m}}+P_{\text {loss as c }}}{U_{\mathrm{m}} I_{\mathrm{m}}}-1 .
\end{aligned}
$$

Testing computations have shown that the final choice of the solution must take into account also the course of the electric field intensity near the beginning. As shown below, some combinations could lead to unacceptable high values of the electric field intensity at the end of the near-cathode layer $E(s)$.

\section{Searching Minimums of the OBJective Function}

In this section, exemplary results are given for the following experimental arrangement. The arc heater geometry is characterized by the anode channel radius $r_{\mathrm{C}}=8 \mathrm{~mm}$ and the total length $z_{\mathrm{L}}=109 \mathrm{~mm}$ including the separately cooled segments as follows: the input part $22 \mathrm{~mm}$, the main channel part $60 \mathrm{~mm}$ and the grounded anode $27 \mathrm{~mm}$ long, all with the same diameter. The device was operated on argon, with a rather high gas flow rate of $22.5 \mathrm{~g} / \mathrm{s}$, and with an input power of $18.4 \mathrm{~kW}\left(I_{\mathrm{m}}=162.3 \mathrm{~A}, U_{\mathrm{m}}=113.4 \mathrm{~V}\right)$.

Using the results of preparative calculations, the computational cost of the simplified model of the arc is not extremely high and the expected ranges of the state variables seem not to be too wide. Thus, the easiest and the most illustrative way of searching the minimum values of $\Delta_{\text {tot }}$ is based on computations of $\Delta_{\text {tot }}$ in a sufficiently dense mesh of all the state variables as presented in Figure 2 Testing computations have shown that the following steps seem to be suitable in the discussed case: 0.01 for the exponent $n_{\mathrm{r}}$, $0.01 \times 10^{8} \mathrm{Am}^{-2}$ for the current density at the cathode tip $j_{0}$, and $10 \mathrm{~K}$ for the temperature at the end of the near-cathode layer $T_{\mathrm{A}}(s)$. In Figure 2 the obtained results of $\Delta_{\text {tot }}$ (in percentages) are presented for seven values of $n_{\mathrm{r}}$ and suitable ranges of $j_{0}$ and $T_{\mathrm{A}}(s)$. 


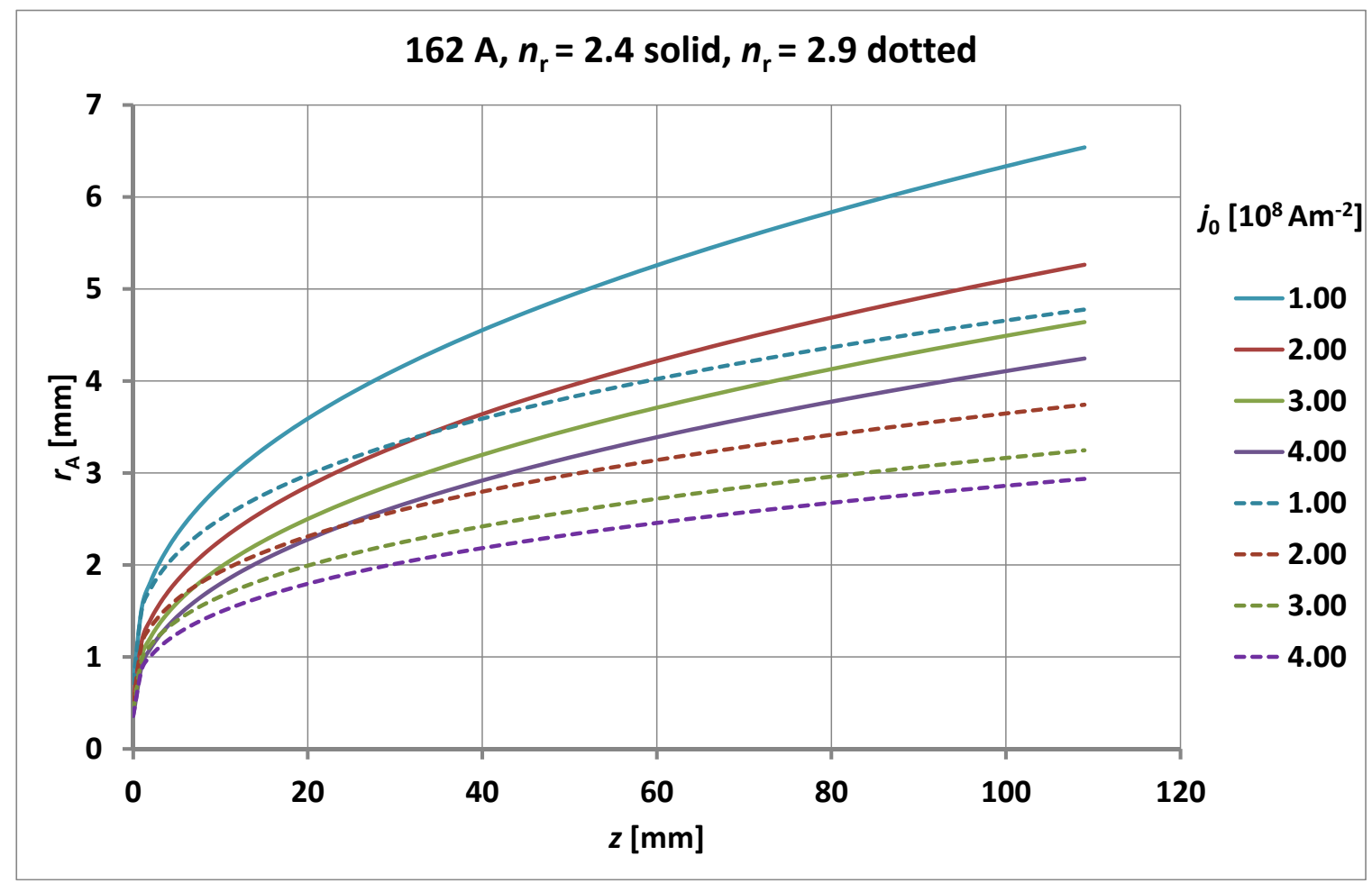

Figure 1. The influence of the choice of the exponent $n_{\mathrm{r}}$ and the current density on the cathode tip $j_{0}$ on the arc radius along the anode channel. Exemplary calculation (1) for the arc current $I=162 \mathrm{~A}$ and the anode channel length $z_{\mathrm{L}}=109 \mathrm{~mm}$.

For each exponent $n_{\mathrm{r}}$, two figures are shown. In the $3 \mathrm{D}$ diagram, the total shape of the surface and especially its steep or flat regions can be observed. In its projection to $\left[T_{\mathrm{A}}(s) j_{0}\right]$ plane, the region with the minimum value of $\Delta_{\text {tot }}$ can be identified, altogether with the corresponding range of both state variables. Below each pair of figures, the found minimum value is given with the corresponding vector of the state variables.

To make figures clear and easy to read, only the chosen regions around the minimums are depicted in each part of Figure 2 For the same reason, the parts where $\Delta_{\text {tot }}$ exceeds $25 \%$ are cut off (light blue areas).

Obviously, in the tested experiment, with a low exponent $n_{\mathrm{r}}=2.40$ the surface $\Delta_{\text {tot }}\left(T_{\mathrm{A}}(s), j_{0}\right)$ is rather flat and the values of $\Delta_{\text {tot }}$ are above $10 \%$. With the increasing exponent $n_{\mathrm{r}}$, first, a region with $\Delta_{\text {tot }}$ lower than $10 \%$ appears and the further increase of the exponent $n_{\mathrm{r}}$ leads to its splitting into two local minimums. Simultaneously, these two zones are becoming smaller, and for $n_{\mathrm{r}}$ above $2.90, \Delta_{\text {tot }}$ is above $10 \%$ again. The best solution was found for $[2.57$; $\left.2.57 \times 10^{8} \mathrm{Am}^{-2} ; 12080 \mathrm{~K}\right]$ with $\Delta_{\text {tot } \min }=8.90 \%$.

\section{Results And Discussion}

For better understanding of the mutual relations between the state variables and other important quantities in the region of interest, minimum values of $\Delta_{\text {tot }}$ were found for many values of $n_{\mathrm{r}}$ between 2.30 and 4.00 .
The results are summarized in Figure 3 Altogether with the curve $\Delta_{\text {tot min }}$ vs. $n_{\mathrm{r}}$, also dependences of the current density on the cathode tip $j_{0}$, the arc temperature at the end of the near-cathode layer $T_{\mathrm{A}}(s)$, the computed width of the near-cathode layer $s$, the computed arc temperature $T_{\mathrm{A}}\left(z_{1}\right)$ at the first step $\Delta z$ ( $1 \mathrm{~mm}$ here) from the beginning, and the computed electric field intensity at the end of the near-cathode layer $E(s)$ on the exponent $n_{\mathrm{r}}$ are depicted in Figure 3 . (To make the figure easy to read, only a half of $E(s)$ is given in Figure 3 )

As can be seen in Figure 3, with the increasing $n_{\mathrm{r}}$, the curve $\Delta_{\text {tot min }}$ vs. $n_{\mathrm{r}}$ first decreases rather quickly, exhibits a minimum near $n_{\mathrm{r}}=2.50$ and then permanently slowly increases.

The corresponding current density $j_{0}$ at the cathode tip permanently slightly decreases. The same is true also for the third state variable, the arc temperature at the end of the near-cathode layer $T_{\mathrm{A}}(s)$, but here, a mutual comparison to the computed arc temperature $T_{\mathrm{A}}\left(z_{1}\right)$ at the first step $\Delta z$ from the beginning is worth mentioning. For a low $n_{\mathrm{r}}$, the computed arc temperature $T_{\mathrm{A}}\left(z_{1}\right)$ at the first step $\Delta z$ from the beginning is significantly lower than the arc temperature at the end of the near-cathode layer $T_{\mathrm{A}}(s)$. The curves $T_{\mathrm{A}}(s)$ vs. $n_{\mathrm{r}}$ and $T_{\mathrm{A}}\left(z_{1}\right)$ vs. $n_{\mathrm{r}}$ intersect for $n_{\mathrm{r}}=2.57$, near above the global minimum of $\Delta_{\text {tot }}$, and then the curve $T_{\mathrm{A}}\left(z_{1}\right)$ vs. $n_{\mathrm{r}}$ increases and reaches much higher values than $T_{\mathrm{A}}(s)$ vs. $n_{\mathrm{r}}$. Obviously, the requirement of a steep and non-oscillating increase of the arc temperature near the beginning can be met 


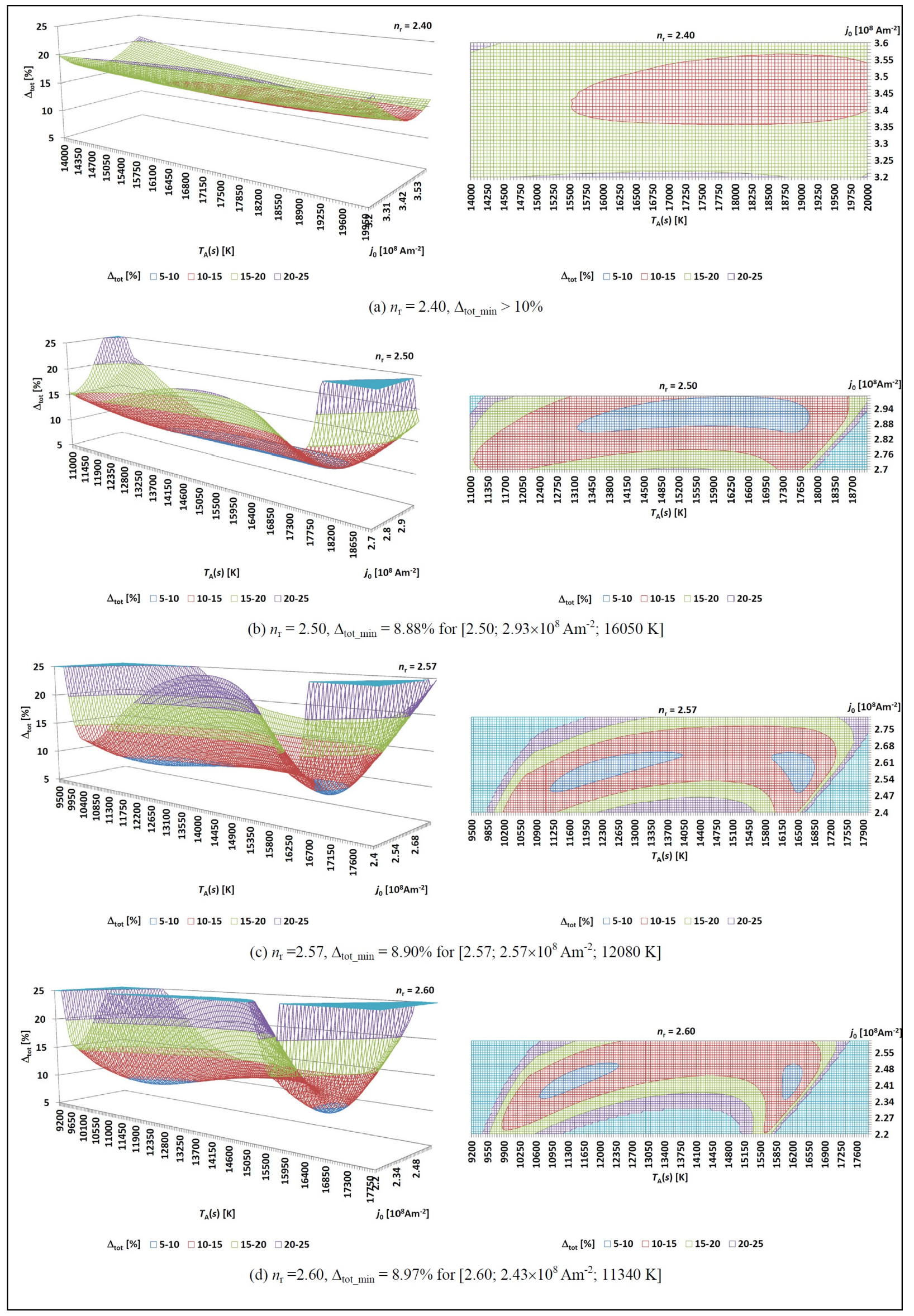

figure continues on the next page 
continued

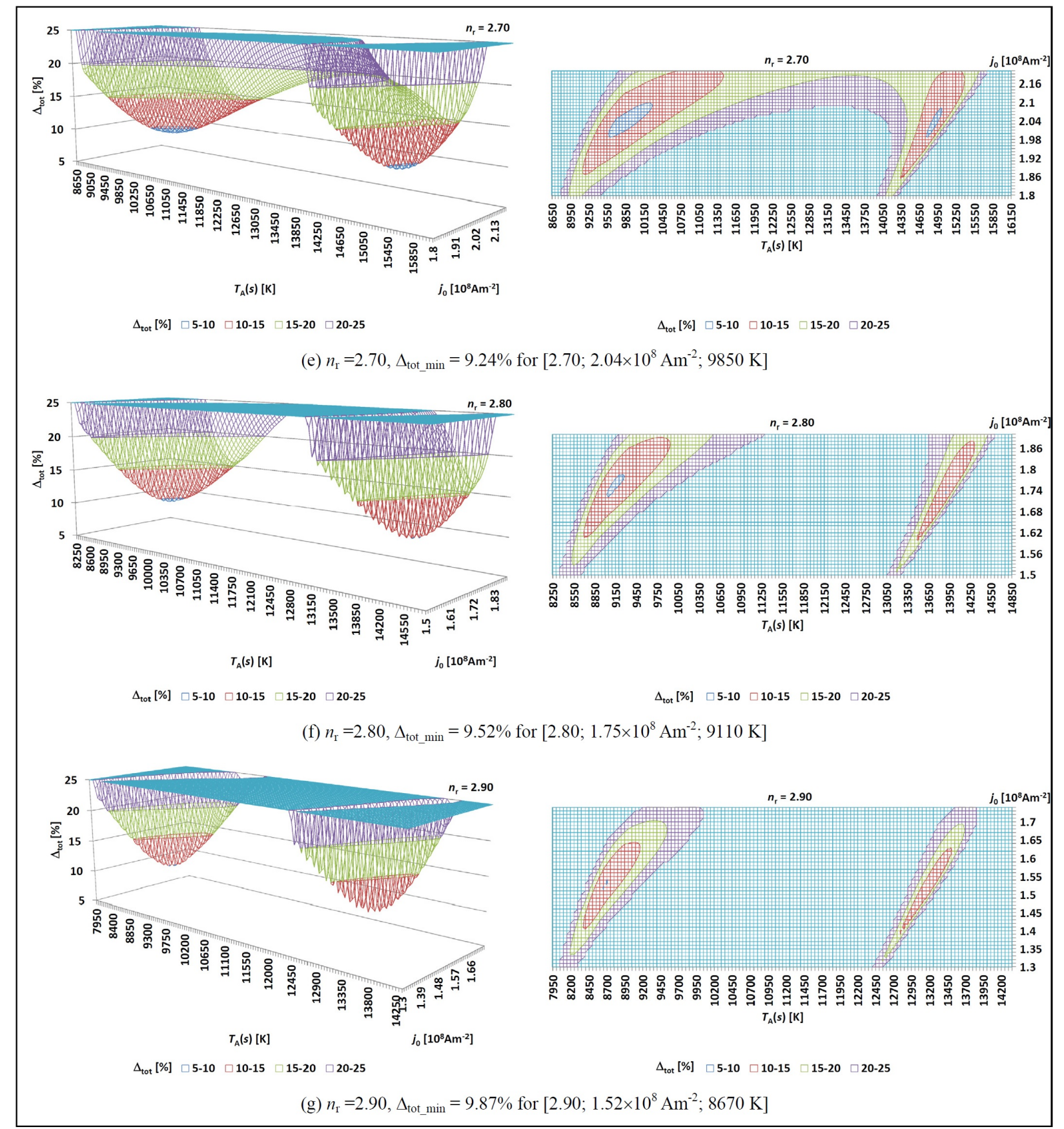

Figure 2. The course of the objective function $\Delta_{\text {tot }}$ for the chosen values of the state quantities $\left[n_{\mathrm{r}}[-] ; j_{0}\left[10^{8}\right.\right.$ $\left.\left.\mathrm{Am}^{-2}\right] ; T_{\mathrm{A}}(s)[\mathrm{K}]\right]$ in the regions of interest and the reached minimum values of the objective function $\Delta_{\text {tot min }}$; the example for the arc current $I=162 \mathrm{~A}$ and argon flow rate $22.5 \mathrm{~g} / \mathrm{s}$. Legend: $\Delta_{\text {tot }}$ between 5 and $10 \%$ dark blue, between 10 and $15 \%$ red, between 15 and $20 \%$ green, between 20 and $25 \%$ violet, above $25 \%$ light blue, cut off.

just in the region between $n_{\mathrm{r}}=2.50$ and 2.60 , close above the global minimum of $\Delta_{\text {tot }}$.

Similarly, the computed width of the near cathode layer $s$ remains almost constant between $n_{\mathrm{r}}=2.30$ and $n_{\mathrm{r}}=2.50$ where its value agrees well with an experimentally obtained width of near-cathode layer given by other authors [8]. With a further increase of $n_{\mathrm{r}}$, the computed width of the near cathode layer $s$ decreases.

A significant change near the minimum of $\Delta_{\text {tot min }}$ can be observed also for the curve of $E(s) / 2$ in Fig- ure 3 First, the computed electric field intensity $E(s)$ is around $4 \mathrm{kV} / \mathrm{m}$, but when the exponent $n_{\mathrm{r}}$ exceeds the value of 2.50 (the global minimum of $\Delta_{\text {tot }}$ ), it starts to grow and reaches unacceptable values of $80 \mathrm{kV} / \mathrm{m}$.

Figure 4 shows again the dependence of the minimum values of the objective function $\Delta_{\text {tot min }}$ on the exponent $n_{\mathrm{r}}$, but simultaneously, also three constituent parts of $\Delta_{\text {tot }}$ are given. Their mutual comparison reveals the reason why the global minimum of $\Delta_{\text {tot }}$ is reached. For exponent $n_{\mathrm{r}}=2.30$, both $\Delta_{\text {in }}$ 


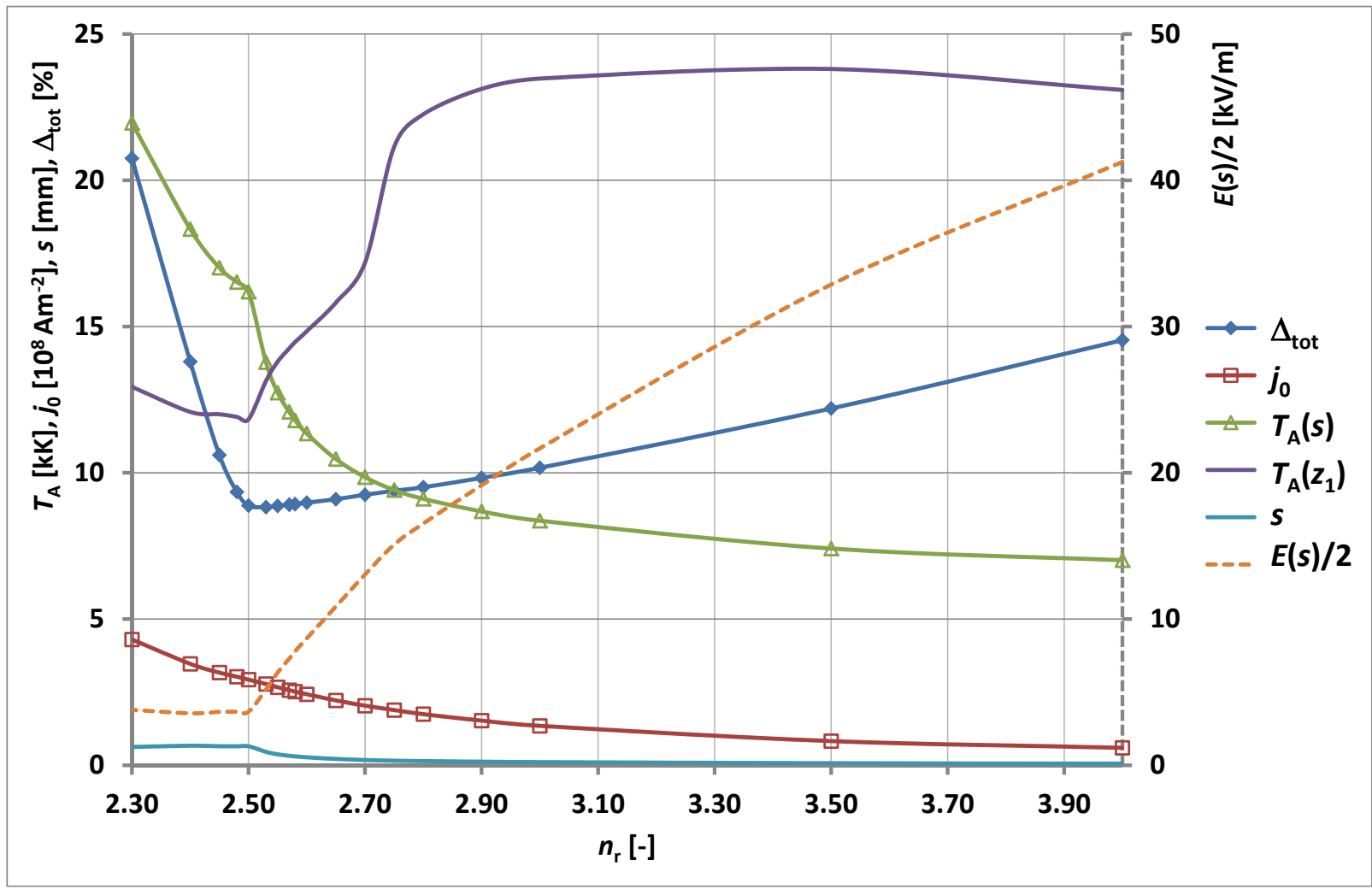

FigURE 3. The found minimums of the objective function $\Delta_{\text {tot min }}$, altogether with the corresponding values of the other state quantities $j_{0}$ and $T_{\mathrm{A}}(s)$, their related limiting quantities $T_{\mathrm{A}}\left(z_{1}\right)$ and $E(s)$ and the computed width of the near-cathode layer $s$, all vs. exponent $n_{\mathrm{r}}$; the example for the arc current $I=162 \mathrm{~A}$ and argon flow rate $22.5 \mathrm{~g} / \mathrm{s}$.

and $\Delta_{\mathrm{ch}}$ are rather high and their sign is opposite. For $n_{\mathrm{r}}=2.53$, both come down almost to zero and remain near the zero value up to $n_{\mathrm{r}}=3.50$, when they seem to start increasing, with opposite signs again. The third component of the objective function, $\Delta_{\mathrm{U}}$, prevails the other two almost for all tested $n_{\mathrm{r}}$. It permanently grows, slower at a lower $n_{\mathrm{r}}$. This third constituent part has the biggest share in the total objective function and in fact, it determines its value, but where the minimum value of the total objective function is reached depends mainly on other two components, $\Delta_{\text {in }}$ and $\Delta_{\text {ch }}$.

\section{Conclusions}

The paper deals with the updated version of the simplified model of an intensively blasted electric arc burning in argon in the anode channel of the modulartype experimental arc heater. Previous computations and experiments have revealed the necessity of completing the set of the state variables with the current density at the cathode tip, and also of setting limiting conditions not only for the temperature but also electric field intensity at the end of the near-cathode layer. To demonstrate the impact of the choice of individual parameters and the share of components in the reached minimum objective function, the results of the modified model applied with an exemplary set of real measured data are given in figures. In the presented example, the values of the exponent deter- mining the arc radius development along the anode channel axis, the current density at the cathode, and the arc temperature at the end of the near-cathode layer, similarly as the computed width of the nearcathode layer match the data given by other authors. Whether the procedure works well under various operational conditions of the arc heater or still needs some refinement, must be tested and verified by further computations with different sets of experimental data.

\section{ACKNOWLEDGEMENTS}

This research work has been carried out in the Centre for Research and Utilization of Renewable Energy (CVVOZE). Authors gratefully acknowledge financial support from the Ministry of Education, Youth and Sports of the Czech Republic under NPU I programme (project No. LO1210 Energy for Sustainable Development).

\section{REFERENCES}

[1] J. Senk, I. Jakubova, I. Laznickova. Analysis of intensively blasted electric arc burning in the arc heater's anode channel. Acta Polytechnica 56(5):395-401, 2016. DOI:10.14311/AP.2016.56.0395

[2] J. Senk, I. Jakubova, I. Laznickova. Treatment of near-electrode regions in a simple model of blasted electric arc. In Proceedings of EPE 2016. Praha, p. 426-431, 2016. DOI:10.1109/EPE.2016.7521749.

[3] J. Lowke. Predictions of arc temperature profiles using approximate emission coefficients for radiation 


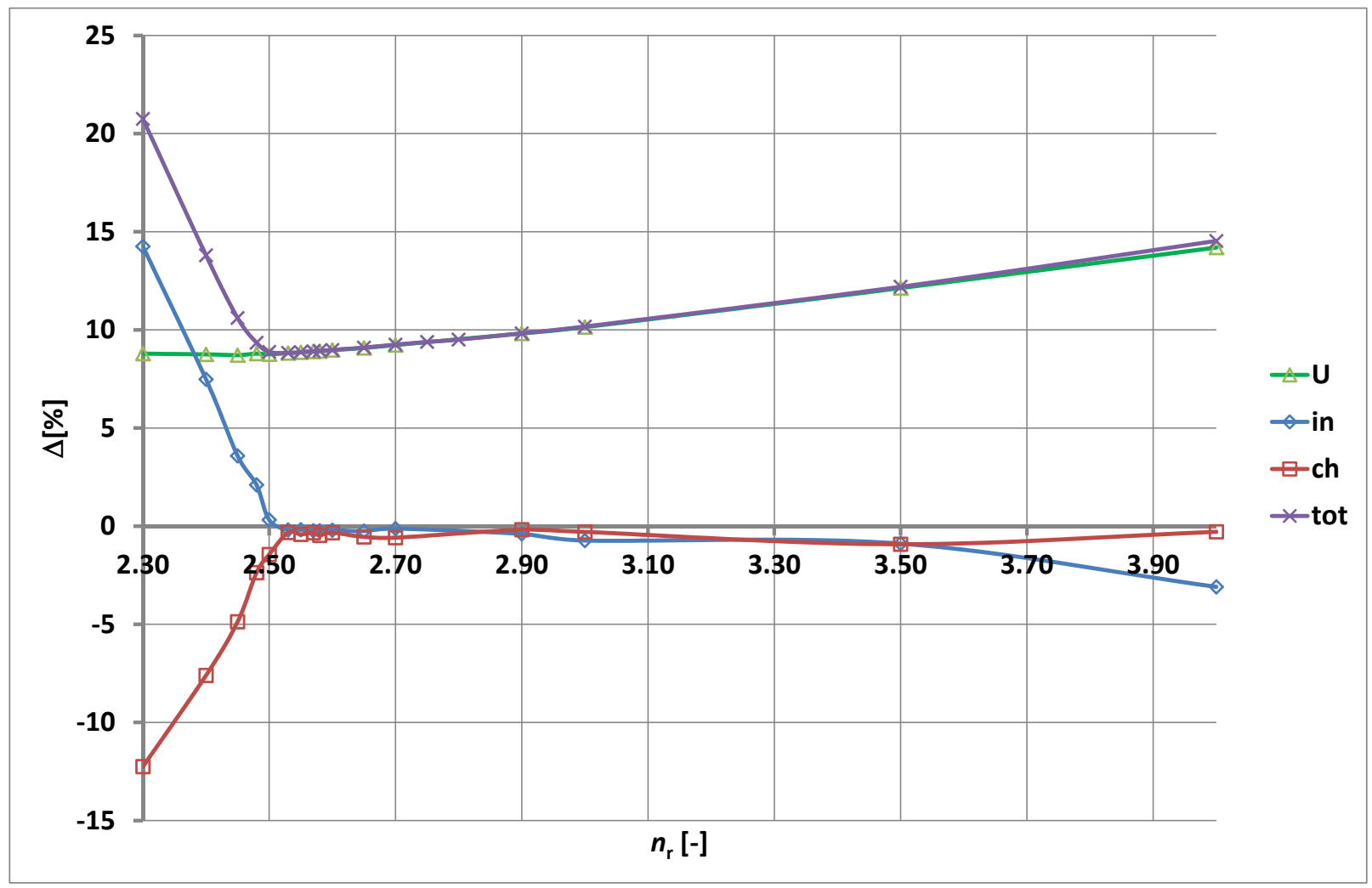

FigURE 4 . The found minimums of the objective function $\Delta_{\text {tot min }}$ and the corresponding individual constituent parts of $\Delta_{\text {tot min }}\left(\Delta_{\text {in min }}, \Delta_{\text {ch min }}\right.$ and $\left.\Delta_{\mathrm{U} \text { min }}\right)$ vs. exponent $n_{\mathrm{r}}$; the example for the arc current $I=162 \mathrm{~A}$ and argon flow rate $22.5 \mathrm{~g} / \mathrm{s}$.

losses. J Quant Spectrosc Radiat Transfer 14:111-122, 1974. DOI:10.1016/0022-4073(74)90004-1.

[4] J. Senk, I. Laznickova, I. Jakubova. Power loss distribution along the arc heater with intensively blasted electric arc. In Proceedings of EPE 201\%. Technical Univ. of Ostrava, 2017. DOI:10.1109/EPE.2017.7967257

[5] P. Kloc. Computed values of net emission coefficient of argon. Private communication, 2018.

[6] H. Schlichting. Grenzschicht Theorie. Verlag G. Braun, Karlsruhe, Germany, 1965.
[7] S. Ramakrishnan, A. Stokes, J. J. Lowke. An approximate model for high-current free-burning arcs. $J$ Phys D: Appl Phys 11:2267-2280, 1978. DOI:10.1088/0022-3727/11/16/014.

[8] A. A. Belevtsev, S. V. Goryachev, E. K. Isakaev, F. V. Chinov. Experimental study on the near-electrode plasma-tungsten cathode system in high-current athmospheric-pressure nitrogen arcs. High Temperature 51(5):583-593, 2013. DOI:10.1134/S0018151X13050027

[9] A. Bauer. Zur Theorie des Kathodenfalls in Lichtbogen. Z Physik 138:35-55, 1954. DOI:10.1007/BF01365533 